\title{
Anorexia Nervosa and Obesity are Associated with Opposite Brain Reward Response
}

\author{
Guido KW Frank', ${ }^{1,2}$ Jeremy R Reynolds ${ }^{3}$, Megan E Shott' ${ }^{1}$, Leah Jappe ${ }^{4}$, Tony T Yang ${ }^{5}$, Jason R Tregellas ${ }^{1,2}$ \\ and Randall C O'Reilly ${ }^{6}$
}

'Department of Psychiatry, University of Colorado, Anschutz Medical Campus, Aurora, CO, USA; ${ }^{2}$ Department of Neuroscience, University of Colorado, Anschutz Medical Campus, Aurora, CO, USA; ${ }^{3}$ Department of Psychology, University of Denver, Denver, CO, USA; ${ }^{4}$ Department of Psychology, University of Minnesota, Minneapolis, MN, USA; ${ }^{5}$ Department of Psychiatry, University of California San Diego, San Diego, CA, USA; ${ }^{6}$ Department of Psychology and Neuroscience, University of Colorado Boulder, Boulder, CO, USA

\begin{abstract}
Anorexia nervosa (AN) is a severe psychiatric disorder associated with food avoidance and malnutrition. In this study, we wanted to test whether we would find brain reward alterations in AN, compared with individuals with normal or increased body weight. We studied 21 underweight, restricting-type AN (age M 22.5, SD 5.8 years), 19 obese (age M 27. I, SD 6.7 years), and 23 healthy control women (age M 24.8, SD 5.6 years), using blood oxygen level-dependent functional magnetic resonance brain imaging together with a rewardconditioning task. This paradigm involves learning the association between conditioned visual stimuli and unconditioned taste stimuli, as well as the unexpected violation of those learned associations. The task has been associated with activation of brain dopamine reward circuits, and it allows the comparison of actual brain response with expected brain activation based on established neuronal models. A group-by-task condition analysis (family-wise-error-corrected $P<0.05$ ) indicated that the orbitofrontal cortex differentiated all three groups. The dopamine model reward-learning signal distinguished groups in the anteroventral striatum, insula, and prefrontal cortex $(P<0.00$ I, 25 voxel cluster threshold), with brain responses that were greater in the AN group, but lesser in the obese group, compared with controls. These results suggest that brain reward circuits are more responsive to food stimuli in AN, but less responsive in obese women. The mechanism for this association is uncertain, but these brain reward response patterns could be biomarkers for the respective weight state.

Neuropsychopharmacology (2012) 37, 203 I-2046; doi:I0.1038/npp.2012.5I; published online 2 May 2012
\end{abstract}

Keywords: anorexia nervosa; obesity; fMRl; dopamine; reward; brain activation

\section{INTRODUCTION}

Anorexia nervosa (AN) is a severe psychiatric disorder that is associated with self starvation and severe fear of weight gain (American Psychiatric Association, 2000). Little is understood about brain pathways that drive those behaviors, but the brain reward system has been proposed as an important candidate for AN research (Halmi, 2009; Kaye et al, 2009). That system drives the motivation to eat, which is related to the neurotransmitter dopamine (Berridge, 2009; Kelley et al, 2005).

Food restriction and weight loss have been associated with heightened brain dopamine-related reward response in rodents (Avena et al, 2008; Carr et al, 2003; Carr, 2007). Over-consumption of food on the contrary showed

\footnotetext{
*Correspondence: Dr GKW Frank, The Children's Hospital, Gary Pavilion A036/B-130, 13123 East 16th Avenue, Aurora, CO 80045, USA, Tel: + I 720777 1909, Fax: + I 7207777306 ,

E-mail: Guido.Frank@ucdenver.edu

Received I5 November 2011; revised 15 March 2012; accepted 16 March 2012
}

addiction-like dopamine D2 receptor downregulation in rodents in brain reward regions (Johnson and Kenny, 2010). Those animal studies suggest that food restriction may sensitize, whereas excessive food intake may desensitize brain reward pathways. Human brain imaging studies indicated that obese individuals have reduced brain response in response to food receipt (Gearhardt et al, 2011; Stice et al, 2010) and reduced brain dopamine receptor availability (Volkow et al, 2008). Those studies support the notion that abnormally high body weight is associated with altered brain function that may involve dopamine pathways.

Some research indicated dopamine alterations in AN, such as low brain dopamine metabolites (Kaye et al, 1984). Others found increased eye-blink compared with controls (Barbato et al, 2006), which suggested heightened dopamine sensitivity (Karson, 1983). Recovered AN showed increased dopamine D2/3 receptor availability in the antero-ventral striatum (Frank et al, 2005), also suggesting higher sensitivity in dopaminergic circuits, but we have little information how such alterations may be functionally important. Functional brain imaging may help bridge that gap. For instance, in previous AN brain-imaging studies, AN 
individuals were more responsive than controls to images of thin bodies in the ventral striatum, a dopamine and rewardrelated brain region (Fladung et al, 2010). Recovered AN showed reduced brain response to repeated application of sweet taste in the insula and striatum (Wagner et al, 2008), but increased response in the caudate nucleus to randomly given monetary (Wagner et al, 2007) or taste reward stimuli (Cowdrey et al, 2011). Overall, the brain-imaging literature on brain reward function in $\mathrm{AN}$ indicates brain reward system alterations in that disorder.

Studying brain circuits that are related to dopamine models in the context of brain reward function is particularly interesting for several reasons. First, within brain reward circuits, dopamine is critically associated with providing signals regarding the presence and amplitude of rewards (Kelley et al, 2005; Schultz, 2002). Such signals facilitate reinforcement learning (Daw and Doya, 2006), and have been found to code the value of a stimulus (Daw et al, 2011; Jocham et al, 2011), which may even include the metabolic value of food (de Araujo et al, 2010). Second, computer models for dopamine neuron reward activation exist that can be related to human in vivo brain function. Such a model is the temporal difference model (Sutton and Barto, 1998). This model is a theoretical framework for computational reward-learning models that predict neuronal brain dopamine response. This model has been previously tested for unexpected reward receipt and omission in animal studies (Schultz et al, 1997), and later validated for human brain imaging (D'Ardenne et al, 2008; O'Doherty et al, 2003). In brief, dopamine neurons exhibit a phasic burst of activation in response to the presentation of an unexpected rewarding stimulus (the primary, unconditioned reward stimulus (US)). After repeated presentation of an additional arbitrary stimulus (the conditioned stimulus (CS)) preceding the US, the phasic activation of dopamine neurons transfers in time to the presentation of the CS. Thus, the CS elicits a conditioned dopamine response. This conditioned response is thought to reflect a prediction regarding upcoming rewards, so that after presentation of the CS, there is a high likelihood of a reward appearing. As it is thought to be a prediction, such a prediction can be violated. If the CS (and therefore the conditioned dopamine response) is not followed by the expected reward (US), then there is a violation of the prediction; and as a consequence, at the time of expected but omitted reward, there is a decrease in dopamine tone. This relationship between CS and US is termed a 'prediction error', the difference between the value of the reward stimulus received and that predicted.

In this study, we wanted to test the hypothesis that $\mathrm{AN}$ individuals would have increased brain reward response to salient stimuli (Avena et al, 2008; Carr, 2007) compared with healthy controls, whereas the opposite would be true for obese individuals. We chose the computational temporal difference model approach, as it is based on dopamine neuronal function related to the motivation to approach food (Kelley et al, 2005), but less on pleasantness or fear of food, and brain activation can be compared with known brain responses from animal models. Alterations found in $\mathrm{AN}$ and obese women (OB) could be markers of the respective weight state, they could be related to brain dopamine function (Carr, 2002) and propose directions for treatment research.

\section{PATIENTS AND METHODS}

\section{Study Participants}

Twenty-one restricting-type AN individuals who were ill for between 6 months and 20 years (M $6.48 \pm 5.29$ years), 19 OB, who were obese for between 2 and 20 years (M 11.25 \pm 5.75 years), and 23 healthy control women (CW), matched for age (Table 1) participated in the study, which was approved by the Colorado Multiple Institutional Review Board.

AN was defined (American Psychiatric Association, 2000) as underweight below $85 \%$ of weight expected for age and height, severe fear of gaining weight, body image distortion, and a lack of menstrual cycle. All AN individuals restricted food intake to maintain the low body weight, none was of the binge eating/purging type. Obesity was defined as having a body mass index (BMI, weight in $\mathrm{kg} /$ height in $\mathrm{m}^{2}$ ) of $\geqslant 30$. The normal BMI, as per definition by the Centers for Disease Control and Prevention, ranges from 18.5 to 24.9, a body weight between 25 and 29.9 is considered overweight, and obesity starts at a BMI of 30 . Below BMI 18.5 is considered underweight, and AN weights start at below 17.5. Using those criteria, both the $\mathrm{AN}$ as well as the $\mathrm{OB}$ group were severely abnormal using those weight criteria.

After complete study description, all subjects gave written informed consent. AN were within their first 1-2 weeks of either inpatient or partial hospital treatment, fulfilled all diagnostic criteria for the disorder, and had no electrolyte, blood count, or other laboratory abnormalities. OB individuals had no psychiatric disorder and were not taking medication. Study procedures were conducted in $\mathrm{CW}$ and $\mathrm{OB}$ during the first 10 days of the menstrual cycle to keep hormonal variation low (Dreher et al, 2007). Psychiatric diagnoses including AN, or absence of psychiatric disorders in $\mathrm{CW}$ or $\mathrm{OB}$, were established using the structured clinical interview for DSM-IV diagnoses (American Psychiatric Association, 2000) applied by a doctoral level interviewer. Ten AN were taking medication (two individuals selective serotonin reuptake inhibitors (SSRIs) together with an atypical antipsychotic, six taking SSRI medication, two taking an atypical antipsychotic alone). Eight AN had an anxiety and depressive disorder, one AN had a depressive disorder without anxiety. Eleven AN were not on psychoactive medication and had no current comorbid psychiatric disorder. Ten $\mathrm{CW}$ and five $\mathrm{OB}$ took birth control pills.

\section{Psychological Assessments}

Once enrolled, study participants completed the following series of self-assessment questionnaires: (1) Drive for Thinness, Bulimia, and Body Dissatisfaction from the Eating Disorder Inventory-3 (Garner, 2004), (2) Harm Avoidance from the Temperament and Character Inventory (Cloninger et al, 1994); (3) State and Trait Anxiety from the Spielberger State and Trait Anxiety Inventory (Spielberger, 1983); (4) Depression from the Beck Depression Inventory (Beck et al, 1961); (5) Reward and Punishment sensitivity from the Sensitivity to Reward and Punishment Questionnaire (O'Connor et al, 2004).

\section{Taste Perception Test}

To assess response to study solutions as well as general taste sensitivity across groups, we applied the following taste 
Table I Demographic and Behavioral Data

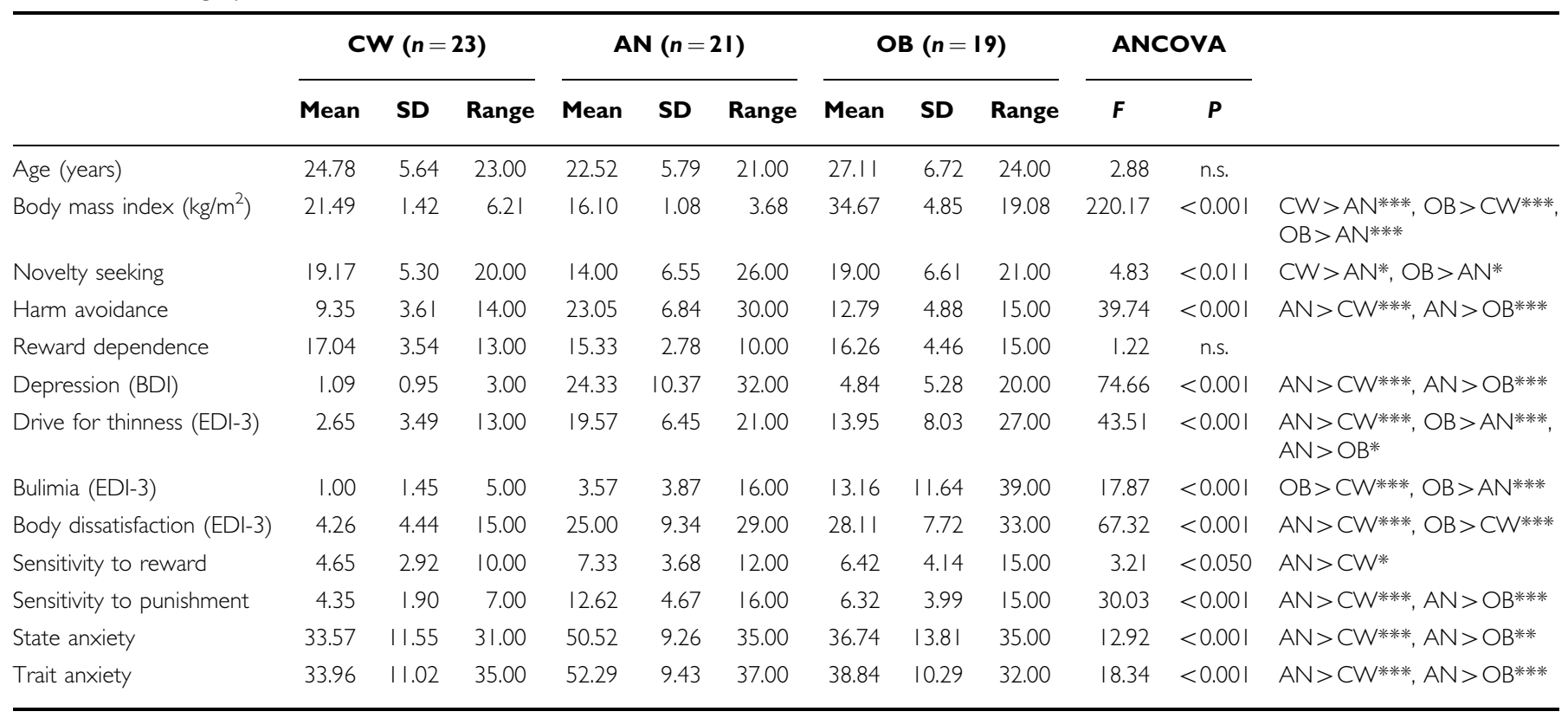

Abbreviations: BDI, Beck Depression Inventory; EDI-3, Eating Disorders Inventory-3.

$* P<0.05, * * P<0.01$, *** $P<0.001$.

perception test. On the morning of the functional magnetic resonance brain imaging (fMRI) scan, before breakfast, subjects were presented with a tray of seven unmarked small cups that contained distilled water, five sucrose solution strengths (Mallinckrodt Chemicals, Phillipsburg, NJ; $2 \%, 4 \%, 8 \%, 16 \%$ and $1 \mathrm{M})$, or Artificial Saliva (25 mM $\mathrm{KCl}, 2 \mathrm{mM} \mathrm{NaHCO}$ ) (O’Doherty et al, 2003). All cups were randomly lined up on the tray, subjects did not know the individual content and rated blindly the solutions for sweetness and pleasantness on 9-point Likert scales. The scales were anchored by the descriptive 'dislike extremely' (1) to 'like extremely' (9) for pleasantness ratings, and 'absent' (1) to 'extreme' (9) for sweetness. The results were analyzed across groups for each taste quality sweetness and pleasantness rating. In addition, we used regression analysis to test (1) whether within groups sweetness or pleasantness ratings across the taste stimuli followed a predictable curve, such as linear, quadratic, and so on, and (2) whether such relationships differed between groups.

\section{Brain-Imaging Procedures}

On the study day between 0700 and $0800 \mathrm{~h}$, AN individuals ate breakfast according to their meal plan, and CW and $\mathrm{OB}$ had a breakfast matched in quality and calories to the average AN meal plan breakfast (calories: CW M $513 \pm 79$, AN M $574 \pm 135$, OB M $567 \pm 132$; p NS). The fMRI imaging was performed between 0800 and $0900 \mathrm{~h}$. Brain images were acquired on a GE Signa $3 \mathrm{~T}$ scanner. $\mathrm{T} 2^{*}$ weighted echo-planar imaging for blood oxygen level-dependent (BOLD) functional activity was performed, voxel size $3.4 \times 3.4 \times 2.6 \mathrm{~mm}$, TR $2100 \mathrm{~ms}$, TE $30 \mathrm{~ms}$, angle $70^{\circ}, 30$ slices, interleaved acquisition, and $2.6 \mathrm{~mm}$ slice thickness with $1.4 \mathrm{~mm}$ gap. We also acquired structural images (T1, SPGR field of view $22 \mathrm{~cm}$, flip angle $10^{\circ}$, slice thickness
$1.2 \mathrm{~mm}$, scan matrix $256 \times 256$, TR 10 , TE 3 , voxel size $1.2 \mathrm{~mm}^{3}$ ) for analysis of brain anatomy.

\section{Classical Conditioning Task}

We adapted the design used by O'Doherty et al (2003). Individuals received three taste stimuli as US during fMRI imaging: $1 \mathrm{M}$ Sucrose solution (100 trials), No solution (100 trials), or Artificial Saliva (80 trials). Individuals learned to associate each taste stimulus with a unique paired visual CS, a geometric shape, which was only probabilistically associated with its corresponding US: the CS shape for No solution was followed in $20 \%$ of the trials by Sucrose (unexpected Sucrose receipt, positive-prediction error condition), and the CS shape for Sucrose was followed in $20 \%$ of trials by No solution (unexpected Sucrose omission, negative-prediction error condition). Each visual cue (CS) was presented for $2 \mathrm{~s}$. With disappearance of the visual cue, simultaneously the taste stimulus (US) was delivered and a black fixation cross appeared on a white background (please see also Supplementary Figure 1). The taste fluid delivery occurred over $1 \mathrm{~s}$. Inter-trial interval was fixed at $6 \mathrm{~s}$. Subjects were instructed to swish their tongue once, look at the fixation cross and await the next trial. For each subject, the first 10 trials were fixed CS shape for sucrose followed by the delivery of US sucrose to establish an initial stable association between the CS sucrose shape and US sucrose taste (O'Doherty et al, 2003). All other trials were fully randomized without predetermined order. The taste stimuli were applied using a customized programmable syringe pump (J-Kem Scientific, St Louis, MO) controlled by E-Prime Software (Psychological Software Tools, Pittsburgh, PA), and individual taste applications were triggered by the MRI scanner's radiofrequency pulse (Frank et al, 2011). Task duration was $28 \mathrm{~min}$. 


\section{Brain-Imaging Analysis}

Brain-imaging data were preprocessed and analyzed using SPM5 software (http://www.fil.ion.ucl.ac.uk/spm/software/ spm5/). Data from each subject were realigned to the first volume, normalized to the Montreal Neurological Institute template, and smoothed with a 3-mm FWHM Gaussian kernel. Each image sequence was manually inspected, and images with artifacts or movement $>$ one voxel size were removed.

Data were modeled with a hemodynamic response function - convolved boxcar function - using the general linear model, including temporal and dispersion derivatives, and autoregression. A $128 \mathrm{~s}$ high-pass filter was applied to remove low-frequency fluctuation in the BOLD signal.

Group-by-condition analysis (analysis 1). We developed first-level models in which we predicted the response in each voxel as a function of each of the five stimulus conditions: expected sucrose, unexpected Sucrose, expected No solution, unexpected No solution, and expected Artificial Saliva. Three contrasts of interest were computed for each subject: (1) trials with the CS for No solution followed by unexpected US Sucrose contrasted against trials with the CS for No solution, followed by expected No solution, positive-prediction error condition; (2) trials with the CS for Sucrose solution followed by unexpected US No solution contrasted against trials with the CS for Sucrose solution, followed by expected Sucrose solution, negativeprediction error condition; (3) the CS that is associated with Sucrose contrasted against the CS associated with No solution, modeled independently from reward receipt, sucrose expectation condition.

Using a random-effects, whole-brain analysis, results were analyzed (1) within groups (family-wise error (FWE)-corrected $P<0.05$, cluster size $\geqslant 25$ voxels), and (2) between groups (group by condition ANCOVA, positiveprediction error condition, negative-prediction error condition, CS Sucrose expectation, FWE-corrected $P<0.05$, cluster size $\geqslant 5$ voxels, with age and depression scores (BDI) as covariates, as depression and age may influence brain reward response (Dreher et al, 2008; Stoy et al, 2011).

We further extracted first-level contrast beta values per subject, based on the SPM group-by-condition significant brain regions to test for the direction of between-group differences.

Computational model analysis (analysis 2). To test temporal difference model-related brain response, we modeled each participant's individual prediction error signal based on trial sequence (O'Doherty et al, 2003; Schultz, 1998). The predicted value $(\widehat{V})$ at any time $(t)$ within a trial is calculated as a linear product of weights $\left(w_{i}\right)$ and the presence of the CS stimulus at time $t$, coded in a stimulus representation vector $x_{i}(t)$, where each stimulus $x_{i}$ is represented separately at each moment in time (O'Doherty et al, 2003):

$$
\widehat{V}(t)=\sum_{i} w_{i} x_{i}(t)
$$

The predicted stimulus value at each time point $t$ in the trial is updated by comparing the predicted value at time $t+1$ to that actually observed at time $t$, leading to the prediction error $\delta(t)$ :

$$
\delta(t)=r(t)+\gamma \widehat{V}(t+1)-\widehat{V}(t)
$$

where $r(t)$ is the reward at time $t$. The parameter $\gamma$ is a discount factor, which determines the extent to which rewards arriving sooner are more important than rewards that arrive later during the task, with $\gamma=0.99$ (O'Doherty et al, 2003). The weights $w_{i}$ relate to how likely a particular US follows the associated CS and are updated on each trial according to the correlation between prediction error and the stimulus representation:

$$
\Delta w_{i}=\alpha \sum_{t} x_{i}(t) \delta(t)
$$

where $\alpha$ is a learning rate. Among various learning rates $(0.2,0.5,0.7)$, a slow $\alpha=0.7$ was the best fit for study groups (O'Doherty et al, 2003). The initial reward values were 1 for Sucrose and 0 for No solution. The trial-to-trial prediction error was then regressed with brain activation across all trials within each subject, and in a second-level random effects analysis compared across groups.

\section{Statistical Analysis}

Behavioral data (ANOVA) and brain-activation beta values (ANCOVA including age and BDI as covariates) were analyzed with SPSS (IBM-SPSS, Chicago, IL) software. For post-hoc group comparison tests, the Scheffe analysis was used when variances across groups were equal and Dunnett's T3 was used when variances were unequal. Pearson's correlation analysis tested behavior-brain response relationships.

\section{RESULTS}

\section{Behavioral Results}

AN individuals had less BMI and scored higher on eating pathology, anxiety, and depression, whereas $\mathrm{OB}$ subjects had increased BMI compared with controls, and also scored higher on eating pathology (Table 1).

Study groups rated $1 \mathrm{M}$ sucrose study solution similarly for pleasantness (CW M $5.3 \pm 2.4$, AN M $4.3 \pm 2.6$, OB M $4.3 \pm 2.5, P \mathrm{NS}$ ) and sweetness (CW M $8.4 \pm 0.8$, AN M $8.8 \pm 0.4$, OB M $8.4 \pm 0.9, P$ NS). Taste sensitivity across the six sucrose concentrations showed significantly positive slopes (linear regression) for sweetness for all three groups, but a condition-by-group analysis was non-significant. For pleasantness, we explored linear, quadratic and logistic regression, but there were no significant relationships in either group.

\section{Brain-Imaging Results}

Whole brain within group analysis of positive-prediction error and negative-prediction error conditions was associated with significant activations in the striatum, insula, orbitofrontal cortex, and amygdala in $\mathrm{CW}$ and $\mathrm{AN}$, whereas OB showed lesser activation in those areas (Table 2a and $b$, 
Table 2 Within- and Between-group Comparison Whole-brain Map Study Results

(a) Receiving reward stimulus unexpectedly, positive-prediction error

Anatomical region

$\boldsymbol{P}<0.05$ FWE-corrected, $\geqslant 25$ voxels

CW

$\mathrm{L}$ amygdala

$\mathrm{L}$ postcentral gyrus

$L$ thalamus

$\mathrm{L}$ thalamus

L cerebellum

$\mathrm{L}$ postcentral gyrus

L parahippocampal gyrus, BA 36

L supplemental motor area, BA 6

$\mathrm{L}$ middle cingulate cortex, BA 31

L supra-marginal gyrus, BA 40

$\mathrm{L}$ temporal pole

L superior temporal cortex, BA 13

$\mathrm{L}$ temporal cortex

$\mathrm{L}$ angular gyrus

$L$ inferior frontal cortex

L superior temporal cortex, BA 42

$\mathrm{L}$ putamen

$L$ frontal operculum

$\mathrm{L}$ precentral gyrus, BA 9

$L$ postcentral gyrus, BA 4

L parietal cortex, BA 40

$\mathrm{L}$ temporal cortex, BA 22

$R$ amygdala

$\mathrm{R}$ rolandic operculum

$R$ postcentral gyrus

$\mathrm{R}$ thalamus

$\mathrm{R}$ anterior cingulate cortex, BA 24

$\mathrm{R}$ parahippocampal gyrus

$\mathrm{R}$ cerebellum

$\mathrm{R}$ lateral orbitofrontal cortex, BA 47

$\mathrm{R}$ inferior frontal cortex

$\mathrm{R}$ frontal operculum

$\mathrm{R}$ head of caudate

$R$ cingulate cortex, BA 24

$\mathrm{R}$ medial orbitofrontal cortex, BA I |

$R$ insula

$R$ insula

AN

L superior frontal gyrus, BA 9

$R$ superior frontal gyrus

$\mathrm{L}$ cingulate cortex, BA 32

$\mathrm{L}$ thalamus

$L$ cuneus, BA 18

L precentral gyrus, BA 6

L occipital cortex

$L$ inferior frontal operculum, BA 44

$L$ thalamus

$R$ calcarine sulcus, BA 18

Cluster voxels

$\mathrm{T} / \mathrm{Z}$ at peak

Location at peak MNI coordinates

$8.41 / 7.70$

$8.04 / 7.41$

$7.72 / 7.16$

$7.32 / 6.83$

$7.16 / 6.70$

$6.88 / 6.47$

$6.84 / 6.43$

$6.79 / 6.40$

$6.78 / 6.39$

$6.73 / 63.4$

6.43/6.09

6.40/6.07

$6.38 / 6.04$

$6.33 / 6.00$

$6.32 / 6.00$

$6.22 / 5.91$

6.16/5.86

6. 14/5.84

$6.13 / 5.83$

$5.99 / 5.71$

5.94/5.67

$5.83 / 5.57$

8.09/7.45

$7.88 / 7.29$

$7.72 / 7.16$

$7.58 / 7.05$

$7.08 / 6.64$

$6.75 / 6.36$

$6.70 / 6.32$

$6.59 / 6.23$

$6.34 / 6.02$

$6.31 / 5.99$

$6.24 / 5.93$

6. $18 / 5.87$

6. 16/5.86

6. 13/5.83

6. $10 / 5.80$

8.33/7.64

8. 10/7.46

$7.99 / 7.38$

$6.79 / 6.40$

6.16/5.86

$6.13 / 5.83$

6. ||/5.8|

$5.96 / 5.68$

5.88/5.6।

$7.85 / 7.26$
$-26,-6,-12$

$-50,-14,36$

$-12,-20,2$

$-14,-14,18$

$-12,-44,-16$

$-58,-4,20$

$-36,-34,-16$

$-10,-2,58$

$-14,-28,42$

$-62,-34,26$

$-60,4,-2$

$-52,-40,20$

$-48,-36,2$

$-46,-52,26$

$-44,28,12$

$-60,-22,10$

$-26,-2,10$

$-40,16,30$

$-52,10,36$

$-30,-32,60$

$-44,-44,44$

$-60,-44,8$

$28,-2,-16$

$48,-10,14$

$54,-8,26$

$10,-16,2$

$6,32,-2$

$28,-24,-22$

$10,-40,-10$

$38,20,-18$

$48,24,18$

$42,14,22$

$10,12,-4$

10, -20, 48

$30,44,-10$

$36,-4,-12$

$32,12,-18$

$-18,54,38$

I, 14, 62

$-8,14,38$

$-6,-26,6$

$-14,-90,20$

$-40,-6,50$

$-38,-80,22$

$-54,6,24$

$-18,-14,2$

2. $-94,10$ 
Table 2 Continued

(a) Receiving reward stimulus unexpectedly, positive-prediction error

\begin{tabular}{|c|c|c|c|}
\hline $\begin{array}{l}\text { Anatomical region } \\
P<0.05 \text { FWE-corrected, } \geqslant 25 \text { voxels }\end{array}$ & Cluster voxels & $\mathbf{T} / \mathbf{Z}$ at peak & Location at peak MNI coordinates \\
\hline \multicolumn{4}{|l|}{ AN (continued) } \\
\hline $\mathrm{R}$ head of caudate & 65 & $7.50 / 6.98$ & $4,8,4$ \\
\hline $\mathrm{R}$ insula & 50 & $6.59 / 6.23$ & $34,-20,4$ \\
\hline R fusiform gyrus & 40 & $6.28 / 5.96$ & $30,-4,-36$ \\
\hline R precentral gyrus, BA 9 & 50 & $6.26 / 5.95$ & $54,6,36$ \\
\hline $\mathrm{R}$ thalamus & 111 & $6.22 / 5.91$ & $10,-12,-2$ \\
\hline $\mathrm{R}$ inferior frontal gyrus & 52 & $6.12 / 5.82$ & $38,16,24$ \\
\hline R precuneus, BA 7 & 33 & $5.96 / 5.68$ & $6,-78,50$ \\
\hline \multicolumn{4}{|l|}{$O B$} \\
\hline L postcentral gyrus, BA 43 & 2126 & $\mid 1.51 / 10.98$ & $-66,-8,16$ \\
\hline L superior temporal cortex & 337 & $8.80 / 8.11$ & $-62,-52,18$ \\
\hline L para-hippocampal gyrus & 80 & $8.32 / 7.63$ & $-24,-40,-10$ \\
\hline L Hippocampus & 71 & $7.59 / 7.06$ & $-30,-14,-12$ \\
\hline L Thalamus & 156 & $7.59 / 7.05$ & $-14,-20,-2$ \\
\hline L Cerebellum & 102 & $7.39 / 6.89$ & $-20,-68,-18$ \\
\hline L posterior cingulate cortex & 76 & $7.08 / 6.64$ & $-12,-44,16$ \\
\hline L inferior parietal cortex, BA 40 & 30 & $7.06 / 6.62$ & $-40,-42,40$ \\
\hline L supplemental motor area, BA 6 & 34 & $6.83 / 6.43$ & $-6,-6,68$ \\
\hline L calcarine sulcus, BA 30 & 75 & $6.79 / 6.39$ & $-18,-58,8$ \\
\hline $\mathrm{R}$ thalamus & 287 & $8.58 / 7.82$ & $10,-20,-2$ \\
\hline R postcentral gyrus & 1592 & $|0.42 / 9.9|$ & $58,-14,38$ \\
\hline R precuneus, BA 30 & 177 & $7.65 / 7.11$ & $4,-52,14$ \\
\hline R superior temporal cortex & 27 & $7.35 / 6.86$ & $64,-32,16$ \\
\hline R amygdala & 78 & $7.23 / 6.76$ & $26,-6,-12$ \\
\hline R precuneus & 59 & $7.23 / 6.76$ & I, $-64,42$ \\
\hline R temporal pole & 47 & $7.22 / 6.76$ & $44,8,-24$ \\
\hline R precuneus, BA 31 & 93 & $7.13 / 6.68$ & $8,-50,36$ \\
\hline $\mathrm{R}$ angular gyrus, BA 39 & 50 & $7.02 / 6.59$ & $52,-62,28$ \\
\hline R postcentral gyrus, BA 3 & 28 & $6.96 / 6.54$ & $30,-36,72$ \\
\hline R fusiform gyrus, BA 37 & 25 & $6.48 / 6.13$ & $36,-58,-20$ \\
\hline $\mathrm{R}$ superior temporal cortex & 28 & $6.17 / 5.87$ & $52,2,-10$ \\
\hline
\end{tabular}

(b) Omission of reward stimulus unexpectedly, negative-prediction error

\begin{tabular}{|c|c|c|c|}
\hline $\begin{array}{l}\text { Anatomical Region } \\
P<0.05 \text { FWE-corrected, } \geqslant 25 \text { voxels }\end{array}$ & Cluster voxels & $T / Z$ at peak & Location at peak MNI coordinates \\
\hline \multicolumn{4}{|l|}{ CW } \\
\hline L postcentral gyrus & 269 & $8.58 / 7.82$ & $-50,-14,36$ \\
\hline $\mathrm{L}$ amygdala & 686 & $7.82 / 7.24$ & $-30,-4,-12$ \\
\hline $\mathrm{L}$ temporal pole & 35 & $7.62 / 7.08$ & $-42,22,-22$ \\
\hline L precuneus, BA 31 & 46 & $7.33 / 6.85$ & $-12,-52,32$ \\
\hline$L$ inferior orbitofrontal cortex & 68 & $7.16 / 6.70$ & $-42,32,-4$ \\
\hline L postcentral gyrus, BA 6 & 62 & $7.03 / 6.60$ & $-62,-2,20$ \\
\hline L temporal cortex & 51 & $6.85 / 6.44$ & $-52,-30,-12$ \\
\hline$L$ temporal cortex & 59 & $6.74 / 6.35$ & $-60,-10,-6$ \\
\hline$L$ lingual gyrus & 77 & $6.63 / 6.26$ & $-14,-62,-12$ \\
\hline L postcentral gyrus & 50 & $6.51 / 6.16$ & $-30,-30,52$ \\
\hline L cuneus & 209 & $6.49 / 6.14$ & $-6,-70,20$ \\
\hline L superior temporal cortex & 184 & $6.48 / 6.14$ & $-54-264$ \\
\hline
\end{tabular}

Neuropsychopharmacology 
Table 2 Continued

(b) Omission of reward stimulus unexpectedly, negative-prediction error

\section{Anatomical Region}

$P<0.05$ FWE-corrected, $\geqslant 25$ voxels

Cluster voxels

$T / Z$ at peak

Location at peak MNI coordinates

\section{CW (continued)}

$L$ thalamus

L cerebellum

$L$ inferior frontal cortex

$\mathrm{L}$ middle cingulate cortex

$L$ precuneus

$\mathrm{L}$ middle cingulate cortex, BA 3 I

$\mathrm{R}$ postcentral gyrus, BA 4

$R$ amygdala

$\mathrm{R}$ thalamus

R temporal cortex, BA 22

$\mathrm{R}$ inferior orbitofrontal cortex, BA 47

$\mathrm{R}$ rolandic operculum

$\mathrm{R}$ cerebellum

$R$ fusiform gyrus

$R$ frontal cortex

$\mathrm{R}$ calcarine sulcus, BA 31

$R$ putamen

$\mathrm{R}$ inferior orbitofrontal cortex

AN

$L$ thalamus

$L$ medial orbitofrontal cortex, BA 32

L superior temporal cortex, BA 13

L supra-marginal gyrus, BA 3

$L$ fusiform gyrus

$\mathrm{R}$ medial frontal cortex, BA 46

$\mathrm{R}$ superior temporal gyrus

$\mathrm{R}$ middle frontal gyrus, BA 10

$R$ cuneus, BA 19

R precentral gyrus, BA 6

$R$ medial frontal cortex, BA 6

R temporal cortex, BA 20

$R$ postcentral gyrus

$R$ fusiform gyrus

$\mathrm{R}$ precuneus, BA 7

$R$ calcarine sulcus, BA 3

$\mathrm{R}$ occipital cortex

$R$ cuneus, BA 19

$\mathrm{R}$ parietal cortex

$\mathrm{R}$ temporal cortex

$O B$

$\mathrm{L}$ postcentral gyrus

$\mathrm{L}$ thalamus

$\mathrm{L}$ hippocampus

$\mathrm{L}$ putamen

$L$ lingual gyrus, BA 18

$\mathrm{L}$ rolandic operculum

$R$ heschl gyrus, BA 42
72

159

27

40

66

38

424

102

151

212

26

58

39

50

40

55

88

28

373

51

52

14 |

51

15097

339

43

538

39

32

58

25

733

42

42

37

32
6.44/6.10

$6.24 / 5.92$

$5.98 / 5.70$

$5.96 / 5.69$

$5.90 / 5.63$

$5.70 / 5.46$

$9.38 / 8.72$

$8.51 / 7.77$

$7.62 / 7.08$

$7.13 / 6.68$

$7.03 / 6.59$

$6.64 / 6.27$

$6.53 / 6.17$

$6.45 / 6.11$

6.44/6.09

$6.35 / 6.02$

$6.29 / 5.97$

6. 15/5.85

$7.46 / 6.95$

$7.11 / 6.66$

$6.94 / 6.52$

$6.89 / 6.48$

$6.37 / 6.03$

$8.90 / 8.42$

$8.72 / 8.36$

$8.63 / 8.27$

$8.19 / 7.53$

$7.89 / 7.29$

$7.4 \mid / 6.91$

$7.19 / 6.73$

$7.15 / 6.69$

$6.55 / 6.19$

$6.55 / 6.19$

$6.54 / 6.18$

$6.26 / 5.94$

$6.24 / 5.93$

$6.09 / 5.79$

$6.07 / 5.78$
$-14,-22,4$

$-12,-50,-14$

$-46,26,6$

$-12,-28,46$

$-2,-54,36$

$-6,-10,46$

$38,-20,40$

$28,-2,-16$

$10,-16,4$

$58,-44,6$

$22,14,-20$

$46,-10,16$

$14,-70,-20$

$36,-10,-28$

$36,8,34$

$12,-68,14$

$24,4,4$

$42,28,-6$

$-14,-16,4$

$-2,46,-4$

$-52,-34,20$

$-60,-16,28$

$-34,-2,-40$

$42,38,24$

$60,-18,6$

$38,36,30$

4, $-94,26$

$32,-12,66$

$32,0,52$

$52,-6,-24$

$28,-4,-36$

$6,-80,50$

$6,-72,14$

14, -76, 42

$-10,-90,32$

$48,-26,40$

$60,-12,-8$
$34,-30,40$
$8.23 / 7.56$

$7.36 / 6.87$

$7.17 / 6.71$

$6.78 / 6.38$

$6.33 / 6.00$

$5.93 / 5.65$

$8.47 / 7.74$
$-54,-8,26$

$-18,-20,4$

$-30,-12,-16$

$-32,-10,-8$

$-20,-78,-12$

$-54,6,8$

$60,-10,10$ 
Table 2 Continued

(b) Omission of reward stimulus unexpectedly, negative-prediction error

\begin{tabular}{|c|c|c|c|}
\hline $\begin{array}{l}\text { Anatomical Region } \\
P<0.05 \text { FWE-corrected, } \geqslant 25 \text { voxels }\end{array}$ & Cluster voxels & $\mathrm{T} / \mathrm{Z}$ at peak & Location at peak MNI coordinates \\
\hline \multicolumn{4}{|l|}{ OB (continued) } \\
\hline R amygdala & 64 & $8.01 / 7.39$ & $24,-2,-16$ \\
\hline R postcentral gyrus, BA 4 & 353 & $7.96 / 7.35$ & $58,-12,32$ \\
\hline R hippocampus, BA 28 & 41 & $7.59 / 7.06$ & $20,-24,-10$ \\
\hline $\mathrm{R}$ thalamus & 72 & $7.38 / 6.89$ & $10,-20,-2$ \\
\hline R temporal pole & 84 & $7.10 / 6.65$ & $38,6,-22$ \\
\hline R temporal cortex, BA 22 & 83 & $6.71 / 6.33$ & $64,-34,6$ \\
\hline R temporal cortex, BA 37 & 27 & $6.11 / 5.82$ & $48,-40,-20$ \\
\hline R precuneus, BA 31 & 25 & $5.87 / 5.61$ & $10,-50,36$ \\
\hline R pallidum, putamen & 27 & $5.69 / 5.45$ & $22,-6,4$ \\
\hline
\end{tabular}

(c) Reward stimulus expectation

\begin{tabular}{|c|c|}
\hline $\begin{array}{l}\text { Anatomical region } \\
P<0.05 \text { FWE-corrected, } \geqslant 25 \text { voxels }\end{array}$ & Cluste \\
\hline \multicolumn{2}{|l|}{$C W$} \\
\hline \multicolumn{2}{|l|}{ L temporal pole } \\
\hline \multicolumn{2}{|l|}{ L parietal cortex, BA 40} \\
\hline \multicolumn{2}{|l|}{ L precuneus, BA 31} \\
\hline \multicolumn{2}{|l|}{ L temporal pole } \\
\hline \multicolumn{2}{|l|}{ L occipital cortex } \\
\hline \multicolumn{2}{|l|}{$L$ temporal cortex } \\
\hline \multicolumn{2}{|l|}{$L$ lingual gyrus } \\
\hline \multicolumn{2}{|l|}{ R cerebellum } \\
\hline \multicolumn{2}{|l|}{$\mathrm{R}$ angular gyrus, BA 39} \\
\hline \multicolumn{2}{|l|}{ R temporal cortex, BA 39} \\
\hline \multicolumn{2}{|l|}{ AN } \\
\hline \multicolumn{2}{|l|}{ L occipital cortex, BA 19} \\
\hline \multicolumn{2}{|l|}{ L lingual gyrus } \\
\hline \multicolumn{2}{|l|}{ L frontal cortex, BA 8} \\
\hline \multicolumn{2}{|l|}{ L occipital cortex } \\
\hline \multicolumn{2}{|l|}{$L$ frontal cortex, BA 46} \\
\hline \multicolumn{2}{|l|}{ L occipital cortex, BA 7} \\
\hline \multicolumn{2}{|l|}{ R occipital cortex } \\
\hline \multicolumn{2}{|l|}{ R occipital cortex, BA 19} \\
\hline \multicolumn{2}{|l|}{ R cerebellum } \\
\hline \multicolumn{2}{|l|}{ R parietal cortex, BA 40} \\
\hline \multicolumn{2}{|l|}{ R temporal pole } \\
\hline \multicolumn{2}{|l|}{ R frontal cortex } \\
\hline \multicolumn{2}{|l|}{ R occipital cortex } \\
\hline R occipital cortex & \\
\hline
\end{tabular}

$O B$

L occipital cortex, BA 18

34

128

60

28

58

76

28

41

90

67

125

42

67

95

84

27

253

117

53

31

39

41

49

26

275

29

244

97

51

74

40
$8.63 / 7.84$

$7.37 / 6.88$

$7.01 / 6.58$

$6.72 / 6.34$

$6.45 / 6.10$

$6.15 / 5.84$

$5.91 / 5.64$

$7.33 / 6.84$

$7.03 / 6.59$

$6.47 / 6.12$

$7.76 / 7.19$

$7.63 / 7.09$

$7.16 / 6.70$

$7.00 / 6.57$

$6.90 / 6.48$

$6.11 / 5.81$

$8.76 / 7.89$

$7.85 / 7.27$

$7.84 / 7.25$

$7.42 / 6.92$

$7.10 / 6.66$

$7.01 / 6.58$

$6.82 / 6.42$

$6.33 / 6.00$

$8.28 / 7.60$

$8.14 / 7.49$

$7.86 / 7.27$

$7.76 / 7.19$

$7.42 / 6.92$

$7.30 / 6.82$

$7.23 / 6.67$
$44,2,-22$

$-50,-50,52$

$-12,-52,32$

$-40,12,-24$

$-42,-62,-12$

$-58,-32,-6$

$-22,-90,-12$

$18,-34,-24$

$-56,-66,28$

$54,-56,12$

$-18,-88,34$

$-24,-94,-8$

$-12,36,58$

$-36,-80,18$

$-54,30,24$

$-22,-74,40$

$24,-92,10$

$40,-76,-10$

$10,-36,-20$

$56,-46,46$

$40,12,-36$

$30,26,52$

$24,-86,20$

$42,-80,34$

$-38,-86,-8$

$-18,-26,-2$

$-24,-34,-18$

$-66,-26,-2$

$-38,-46,38$

$-20,-60,10$

$-64,-42,10$

Neuropsychopharmacology 
Table 2 Continued

(c) Reward stimulus expectation

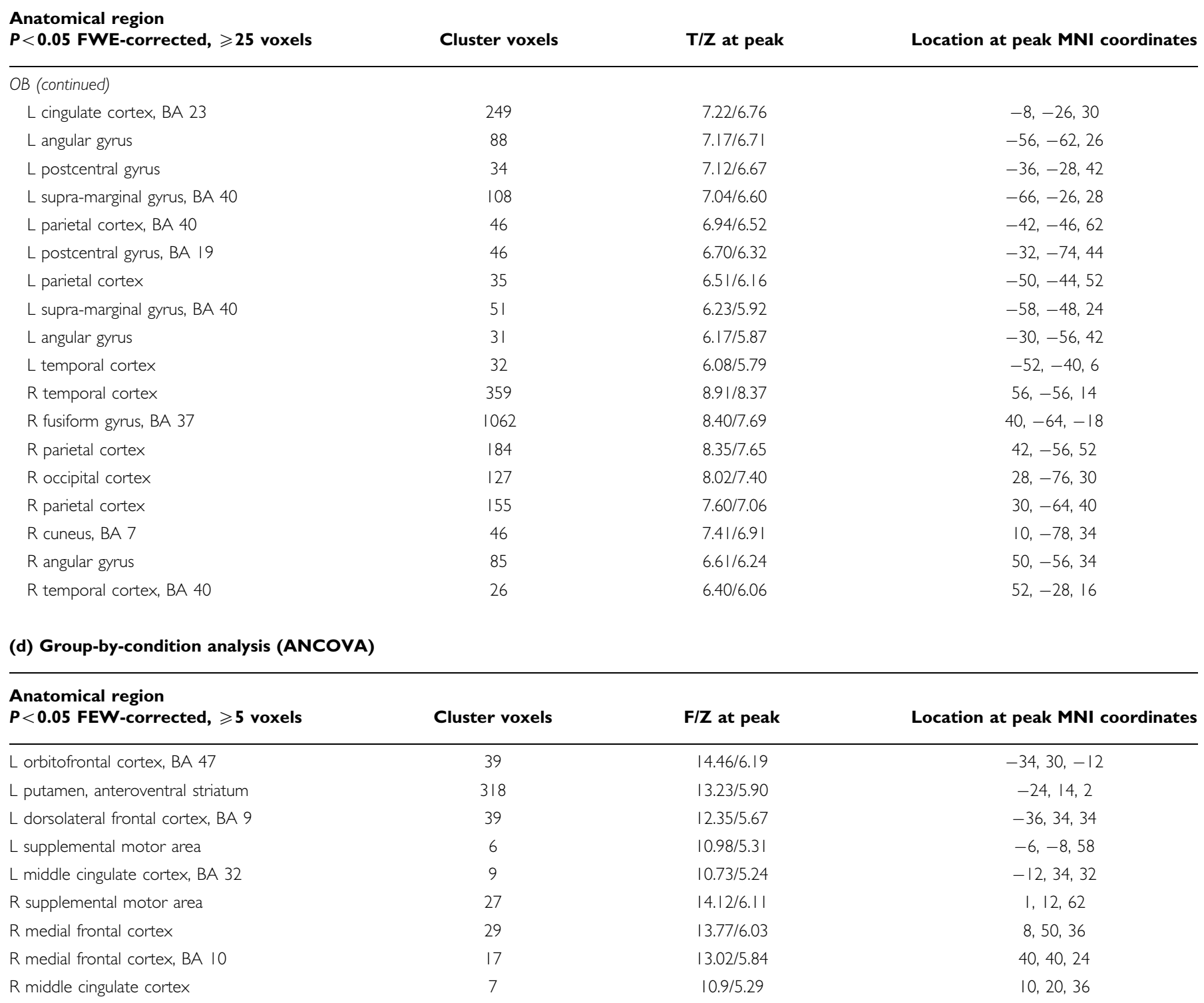

(e) Computational model 3-group ANCOVA

Anatomical region

$P<0.001, \geqslant 25$ voxels

Cluster voxels

$\mathbf{F} / \mathbf{Z}$ at peak

Location at peak MNI coordinates

L prefrontal cortex, BA 8, 9

36

$\mathrm{L}$ hippocampus, thalamus

L dorsolateral prefrontal cortex, BA 9, 46

53

$L$ cingulate cortex, BA 32

$\mathrm{L}$ putamen, anteroventral striatum

L occipital cortex, BA 19

$L$ temporal cortex

$L$ cuneus

R dorsolateral prefrontal cortex, BA 9

$\mathrm{R}$ insula, BA 13

$R$ thalamus

$\begin{array}{ll}36 & 18.83 / 4.77 \\ 53 & 15.56 / 4.37 \\ 498 & 15.32 / 4.34 \\ 78 & 14.32 / 4.20 \\ 99 & 12.02 / 3.85 \\ 33 & 11.58 / 3.78 \\ 27 & 10.83 / 3.65 \\ 39 & 10.62 / 3.61 \\ 634 & 16.37 / 4.48 \\ 26 & 13.58 / 4.09 \\ 27 & 10.22 / 3.54\end{array}$

\section{$-28,42,46$}

$-22,-36,4$

$-38,38,24$

$-2,24,18$

$-20,18,-8$

$-24,-88,36$

$-40,22,-22$

$-18,-96,12$

$40,40,20$

$38,8,-14$

$12,-6,0$ 

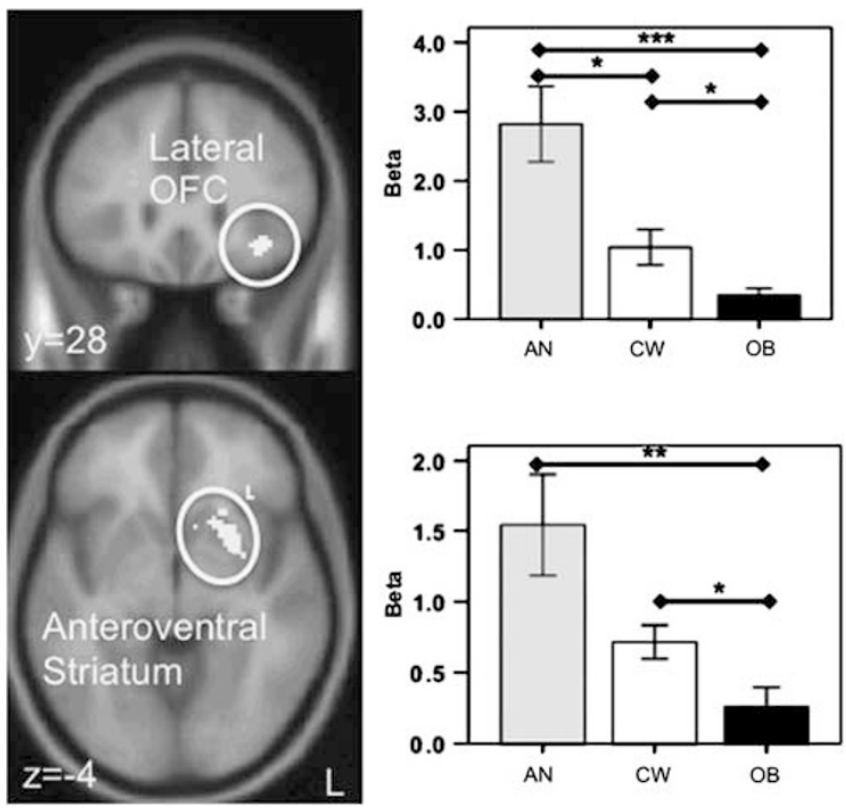

Figure I The group-by-condition analysis indicated significant group differences in the lateral orbitofrontal cortex (OFC), as well as the putamen and anteroventral striatum; brain maps family-wise error (FWE)-corrected $P<0.05$, cluster threshold $\geqslant 5$ voxels. Beta value bar graphs indicated increased OFC response to unexpected reward receipt in anorexia nervosa (AN) compared with controls (CW), but a reduced response in obese women $(\mathrm{OB})$ compared with $\mathrm{CW}$; anteroventral striatum response was reduced in $\mathrm{OB}$ compared with $\mathrm{CW}$ and $\mathrm{AN}$ groups (Dunnett's T3, $* P<0.05$, $* * P<0.0$ I, **** $P<0.00$ I).

Supplementary Figure 2). Sucrose expectation was associated with large activations in temporal, parietal, and occipital cortex in all study groups (Table $2 \mathrm{c}$ ).

Group-by-condition analysis (results analysis 1). The group-by-condition ANCOVA indicated regions of significant difference in the orbitofrontal cortex, the putamen extending into the ventral striatum including nucleus accumbens, cingulate, and prefrontal cortex (Table 2d, Figure 1). The extracted beta values indicated for the positive-prediction error condition, where positive activation is expected (Figure 1, Supplementary Table 1) that all groups were significantly different in the left orbitofrontal cortex, $\mathrm{AN}>\mathrm{CW}>\mathrm{OB}$. For the remaining positive-prediction error condition contrasts, ANs were greater than $\mathrm{OB}$ and than $\mathrm{CW}$ in the right cingulate and right medial frontal cortex, respectively. $\mathrm{CW}$ and $\mathrm{AN}$ were greater than $\mathrm{OB}$ in the left putamen anteroventral striatum and bilateral supplemental motor area. For the negative-prediction error condition, where a relative hypo-activation compared with baseline is expected, ANs were more negative compared with $\mathrm{OB}$ and with $\mathrm{CW}$ in all contrasts, except the left supplemental motor area and left cingulate cortex (Supplementary Table 1).

For Sucrose expectation, only one region showed significant group difference, the right supplemental motor area (Table $2 \mathrm{~d}$, Supplementary Table 1), with CW and OB significantly greater compared with AN.

A limitation of the results is that they could have been confounded by somatosensory stimulation effects from the tongue that could have been different between groups. To address this possibility, we conducted an additional group-by-condition analysis that included the Artificial Saliva trials as control condition. The following contrasts were analyzed:

1. trials with the CS for No solution followed by unexpected US Sucrose, contrasted against trials with the CS for Artificial Saliva, followed by Artificial Saliva (positiveprediction error condition),

2. trials with the CS for Sucrose followed by unexpected US No solution, contrasted against trials with the CS for No solution followed by expected No solution (negativeprediction error condition),

3. the CS that is associated with Sucrose contrasted against the CS associated with Artificial Saliva, modeled independently from reward receipt (Sucrose expectation condition).

The interaction of the group-by-condition analysis $(P<0.05$ FWE-corrected) resulted in 2 significant clusters in the ventral putamen with the following peak voxels: left: $x=-30, y=10, z=-4$, right $x=28, y=-7, z=-4$, cluster size 2 voxels in each ROI. Other significant clusters were in the left orbitofrontal cortex $x=-30, y=30, z=-12$, cluster size 13 voxels; left cingulate cortex $x=-6, y=50, z=4$, cluster size 9 voxels; left medial frontal cortex $x=-26$, $y=56, z=26$, cluster size 40 voxels; right medial frontal cortex $x=12, y=46, z=26$, cluster size 23 voxels. Those clusters are smaller than in the original analysis, but indicate similarly localized regions of group difference after whole-brain correction.

Post-hoc tests (Dunnett's T3) using the extracted beta values from those clusters indicated that for the positiveprediction error condition, AN subjects had greater activation compared with $\mathrm{CW}(P<0.003)$ and $\mathrm{OB}(P<0.001)$ subjects in the right ventral putamen cluster. For the left orbitofrontal cortex cluster, ANs were greater compared with CW $(P<0.05)$ and $\mathrm{OB}(P<0.007)$, and CWs were greater compared with $\mathrm{OB}(P<0.03)$.

For the negative-prediction error condition, AN showed more negative activation compared with $\mathrm{CW}(P<0.007)$ and OB $(P<0.001)$ in the left ventral putamen, as well as for the right ventral putamen $(\mathrm{CW} P<0.05$, OB $P<0.008)$. In the left orbitofrontal cortex, AN had more negative response compared with CW $(P<0.004)$ and $\mathrm{OB}(P<0.003)$.

For sucrose expectation condition, $C W$ showed greater activation compared with $\mathrm{OB}$ in the left prefrontal cortex $(P<0.05)$ and right putamen $(P<0.01)$.

Computational modeling (results analysis 2). Similar to previous results (O'Doherty et al, 2003), the trial-by-trial temporal difference model generated a prediction-errordata-predicted brain activation within groups $(P<0.001$ uncorrected, 25 voxel cluster threshold) in the midbrain and bilateral ventral striatum, and also cingulate cortex, insula, and the orbitofrontal cortex. Between groups, an ANCOVA with age and depression scores as covariates indicated significant group differences in the ventral striatum as well as insula, cingulate, prefrontal, temporal and occipital cortex, and thalamus (Table 2e). The extracted parameter estimates indicated (Figure 2, Table 3) that all groups were significantly different from each other in the left thalamus, left and right dorsolateral frontal cortex, left putamen/ anteroventral striatum, and right insula. 

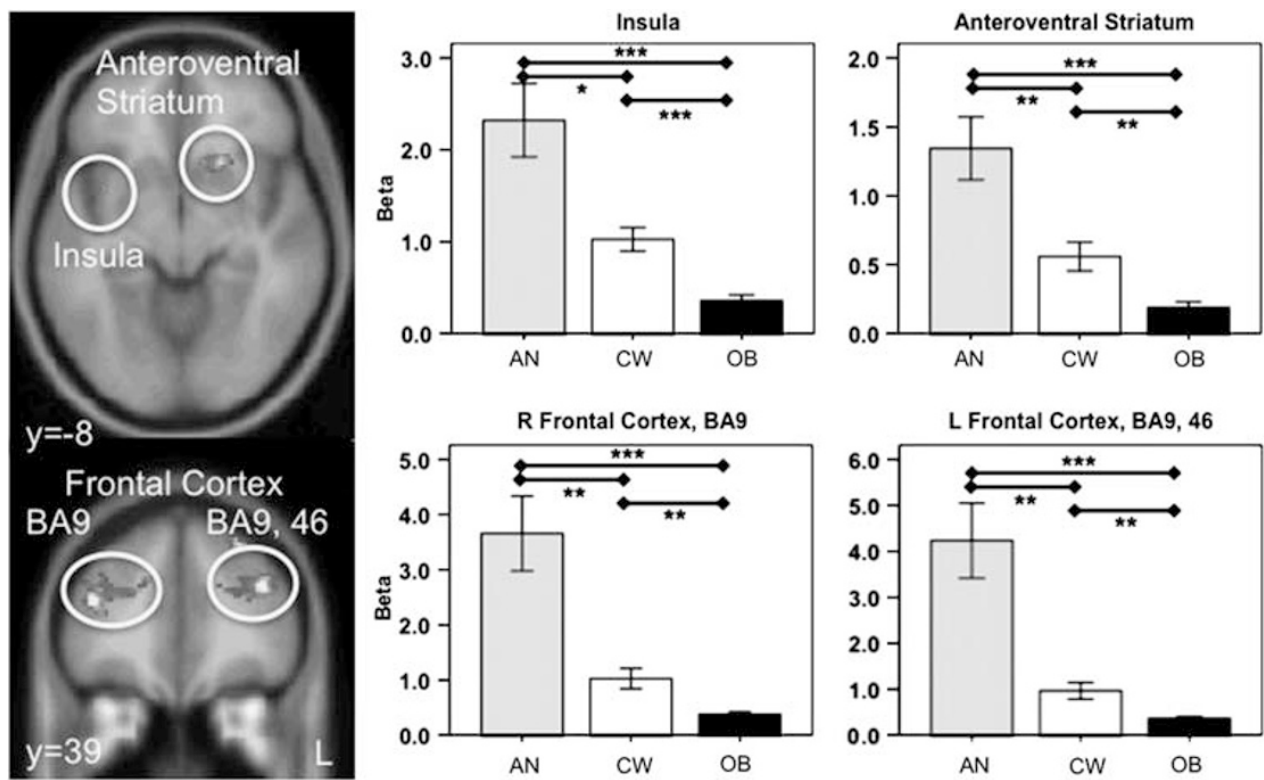

Figure 2 Computational model group comparison (analysis of covariance) indicating greater brain response across groups in the anteroventral striatum, insula, and dorsolateral frontal cortex $(P<0.00$ I uncorrected, cluster threshold $\geqslant 25$ voxels $)$. The extracted parameter estimates for those regions were greater in anorexia nervosa $(\mathrm{AN})$ compared with control $(\mathrm{CW})$, but reduced in obese women $(\mathrm{OB}$; Dunnett's T3, *P $<0.05$, *** $P<0.0 \mathrm{I}$, **** $P<0.001)$

Table 3 Extracted Parameter Estimates (PE) Based on Regions of Group Difference in the Reward-model Brain Response

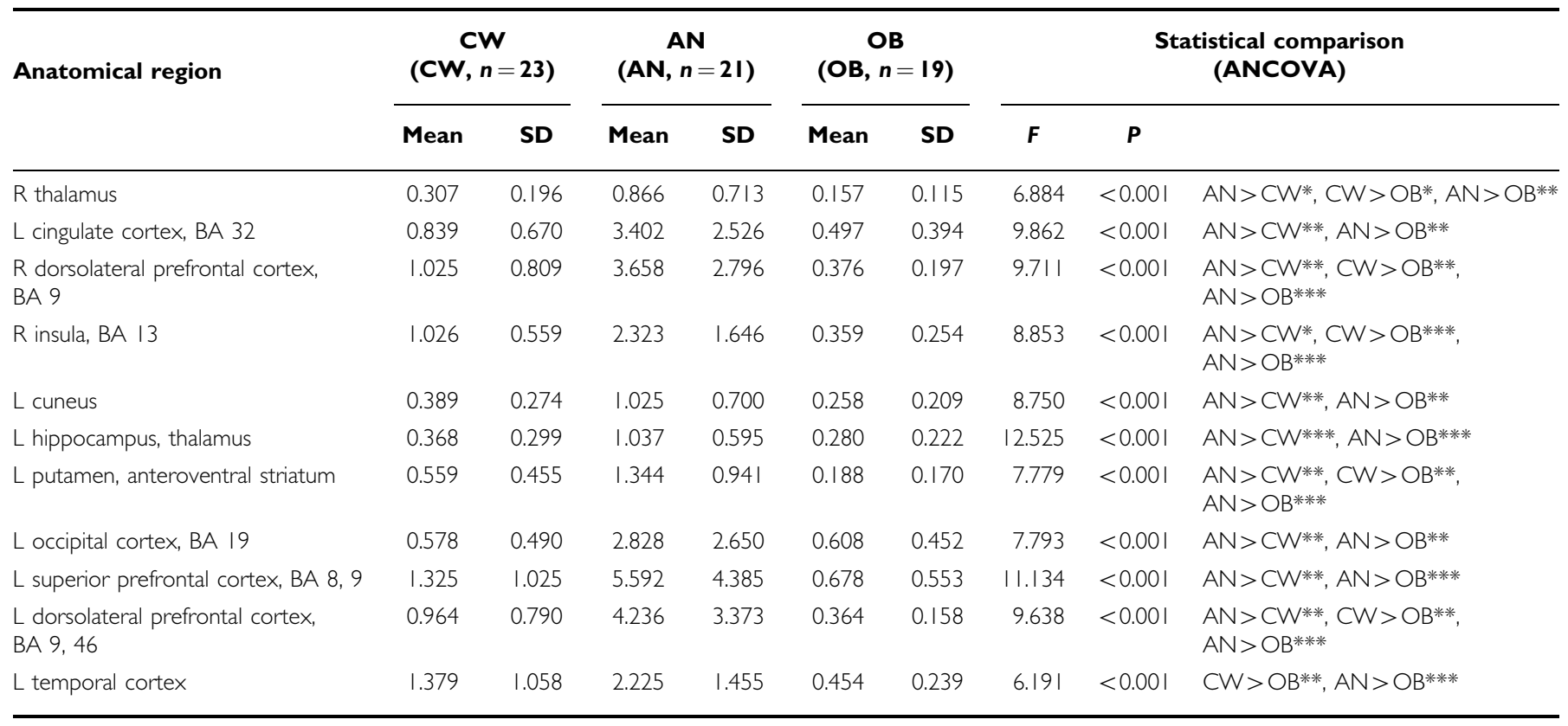

Dunnett's T3, $* P<0.05$, *** $P<0.01$, **** $P<0.001$.

\section{Brain-Imaging Response and Demographic, Behavioral Data}

We explored whether behavioral results for temperament, anxiety or reward response, or duration of illness showed significant relationships with extracted beta values and parameter estimates, but those results were not significantly related to brain-imaging results within the groups.

\section{Effects of Potentially Confounding Variables}

To avoid the effects of comorbid conditions or medication, we compared the 11 unmedicated AN without current depression or anxiety disorder against the $13 \mathrm{CW}$ and $14 \mathrm{OB}$ not on birth control. The group-by-condition ANCOVA indicated $(P<0.001$ uncorrected, 25 voxel cluster threshold) clusters in the bilateral ventral striatum, insula, anterior cingulate, and prefrontal cortex, similar to the full group 
comparison. We further analyzed between the AN subgroups, with vs without medication and comorbid depression or anxiety, whole-brain contrasts (group-by-condition ANCOVA, $P<0.001$ uncorrected, 25 voxel cluster threshold) as well as the beta values ( $t$-test) that had been extracted from the initial group-by-condition ANCOVA. Those analyses did not reveal significant differences across the AN subgroups.

The analysis of group differences for structural gray matter across study groups using SPM and voxel-based morphometry did not show significant group differences $(P<0.05$ FWE-corrected, 25 voxels cluster threshold).

\section{DISCUSSION}

The results of this study indicate that the $\mathrm{AN}$ and $\mathrm{OB}$ individuals show opposite brain reward responses in a taste reward task and using two separate analysis methods. The first analysis, which compared brain response with unexpected reward receipt or omission between groups, suggests that $\mathrm{AN}$ individuals have significantly increased brain activation compared with $\mathrm{CW}$ in the orbitofrontal cortex when receiving reward unexpectedly, whereas $\mathrm{OB}$ have reduced activation in that region compared with CW. The second analysis, which compared known dopamine model neuron response with actual human brain activation to the study task, showed a significantly stronger relationship with the model-derived data in AN vs CW, whereas the $O B$ group response was significantly reduced compared with $\mathrm{CW}$, in the left putamen/anteroventral striatum, right insula, left thalamus, and left and right dorsolateral prefrontal cortex. This suggests that AN may be more and OB less sensitive in dopamine-related pathways compared with CW.

Various studies have investigated reinforcement learning in the context of food intake. Those studies identified brain circuits that involve the ventral striatum, midbrain, insula, orbitofrontal, and anterior cingulate cortex in reward processing (Berridge, 2009; Small et al, 2001). The withingroup results for unexpected receipt or omission of reward stimuli indicated large areas of activation in those regions, but with different intensities across the study groups.

The group-by-condition analysis finding of an opposite group activation in the lateral orbitofrontal cortex in the unexpected reward-receipt condition is important for several reasons. This area was less responsive to food receipt in OB individuals (Gearhardt et al, 2011), and it was hypothesized that lower response in that region could be associated with reduced behavior control and suppression of reward response (Boettiger et al, 2007; Elliott et al, 2000; Goldstein et al, 2007). Reduced lateral orbitofrontal cortex activation in $\mathrm{OB}$ may then be associated with reduced, and increased activation in $\mathrm{AN}$ could be associated with increased impulse control to reward presentation. Thus, increased lateral orbitofrontal cortex activation in AN may be associated with high food-intake control, whereas reduced response in the $\mathrm{OB}$ group could be associated with problems controlling eating. The lateral orbitofrontal cortex has also been associated with learning of stimulus reward associations (Noonan et al, 2010; Tsujimoto et al, 2009), and high activation in the lateral orbitofrontal cortex might indicate high ability to discriminate rewards in $\mathrm{AN}$, but the opposite in $\mathrm{OB}$ individuals.

The computational model regression, that tests how well the in vivo brain response resembles dopamine neuron activation as it is known from dopamine single-neuron recordings, identified the ventral striatum, cingulate, insular and dorsolateral prefrontal cortex, including Brodmann area (BA) 6 bilaterally and BA 46 on the left, as differing across groups. The parameter estimates indicated that all three groups were significantly different from each other in the left putamen/anteroventral striatum and right insula, as well as the left thalamus, and left and right dorsolateral prefrontal cortex. ANs were greater and OBs were lesser compared with CW. Those regions are part of the taste reward system: gustatory inputs from the tongue, immediately after food contact, and before gut involvement, project via brain stem and thalamus to the primary taste cortex comprised of insula and frontal operculum, and from there to the ventral striatum and amygdala, and subsequently to hypothalamus, midbrain, and prefrontal cortex (Carmichael and Price, 1996). In this reward circuitry, DA acts as an important learning signal released in response to unexpected stimuli, but it also drives the motivation to approach food and other rewards, called 'wanting' (Berridge et al, 2010). The same neural pathways that reinforce those natural appetitive behaviors are also activated in response to addictive drugs (Hyman and Malenka, 2001). This has led to the hypothesis that prone individuals could get 'addicted' to food, including increased preference and tolerance, as well as reduction of dysphoria, behaviors that are common in substance-using individuals (Corsica and Pelchat, 2010; Koob and Le Moal, 2005). A recent study using the same design as in this study in bulimia nervosa (BN), an eating disorder with repeated compulsive eating of large amounts of food, showed in fact reduced brain response in the temporal difference model-based analysis compared with controls (Frank et al, 2011). That finding then suggested similarities between $\mathrm{BN}$ and reduced brain response in addiction disorders, supporting the above hypothesis. The results of reduced brain response in the OB group also are consistent with this model of reduced brain reward response in an addiction model of food intake (Gearhardt et al, 2011; Volkow et al, 2008). Altered brain reward function in underweight AN has not been reported before. A recent study in recovered $\mathrm{AN}$ found increased brain response to randomly applied taste stimuli (Cowdrey et al, 2011), whereas others found reduced brain reward response to repeated sweet taste (Wagner et al, 2008). The application of repeated and predictable, $v s$ random and unpredictable taste stimulus receipt most likely accounted for the opposite study results, as unexpected rather than predictable stimulation is related to dopamine activation (Schultz, 2002). The results of our study, together with Cowdrey et al (2011), of heightened brain reward response could be a biomarker of altered brain function in AN, potentially related to brain dopamine. It is unclear whether those alterations are premorbid or develop during the course of illness. The prospective studies in rodents that were exposed to over- or under-consumption of food (Avena et al, 2008; Carr, 2007; Johnson and Kenny, 2010) suggest adaptive dopamine-related changes to food intake, and it is possible that such adaptations also occur in AN and 
OB. Yet, premorbid traits may predispose to such changes. Carr's study (2007) also indicated that brain reward responses persist after weight recovery, which could indicate that alterations in AN during the underweight state persist long into the recovered state, which could be consistent with Cowdrey et al (2011) findings.

A significance of this study is that it is the first that used specifically a dopamine-related reward paradigm in $\mathrm{AN}$, and used computational model-derived data to identify related brain response. Some other studies investigated the dopamine model-related brain response in psychiatric disorders, such as depression (Kumar et al, 2008) or schizophrenia (Waltz et al, 2010), and tended to find reduced brain response in those disorders. Dopamine neuronal reward response can be captured in an algorithm that takes advantage of dopamine neurons responding to unexpected reward stimulus receipt or omission (Schultz, 2002). That model focused originally on the ventral striatum and midbrain, and has recently been expanded to brain regions such as the amygdala, hypothalamus, and cerebellum that are thought to respond to CS, and may drive or inhibit dopamine activation (Hazy et al, 2010). The strong regression between brain response and dopamine model data suggests that the fMRI BOLD response can be associated with brain dopamine activation, although we cannot measure dopamine directly with this technique. Furthermore, the results indicate that a network of brain regions is involved in the brain dopaminerelated reward response, including the cingulate cortex and various prefrontal cortical areas. This circuitry has recently been highlighted in reward and emotional processing in substance use (Volkow et al, 2011), and the connectivity of those regions and how they may affect AN food intake will be an important direction for further study.

Previously, recovered AN had increased dopamine D2/3 receptor availability in the ventral striatum (Frank et al, 2005), but we are not aware of similar studies in an ill group. The dopamine D2 receptor has been associated with brain response to unexpected stimulus omission, whereas the dopamine D1 receptor is thought to mediate response to unexpected reward stimulus receipt (Maia and Frank, 2011). Thus, altered dopamine receptor function could directly affect brain reward response in $\mathrm{AN}$ and $\mathrm{OB}$, but this will require further study using dopamine-specific probes. Research in non-clinical populations suggests this notion. For instance, the dopamine D2 receptor TaqIA A1 gene variant that is associated with reduced receptor density determined food reward response in the brain (Felsted et al, 2010), and genotype of the dopamine transporter predicted appetite suppression in response to stimulant medication (Davis et al, 2007).

Clinically, various dopaminergic drugs have been shown to affect eating and body weight. For instance, stimulants such as methylphenidate or the antidepressant bupropion frequently affect food intake and promote weight loss (Anderson et al, 2002; Goldfield et al, 2007). In AN, small studies using the dopamine D2 antagonist haloperidol or the dopamine D2 partial agonist aripiprazole suggested beneficial effects on core symptoms of AN (Cassano et al, 2003; Trunko et al, 2011). Importantly, the stimulant amphetamine increased, whereas haloperidol decreased brain response in a human temporal difference model paradigm (Menon et al, 2007). In summary, dopaminergic drugs affect food intake, which may be related to brain reward function, supporting that dopaminergic pathways may be involved the pathophysiology of AN and OB.

Only one region, the supplemental motor area, distinguished groups for reward expectation, with a lesser response in the AN group compared with $\mathrm{CW}$ and $\mathrm{OB}$. That region is associated with planning of complex movements and possibly suggests that the AN individuals may prepare less to the upcoming taste stimulus than $\mathrm{CW}$ and $\mathrm{OB}$ groups.

\section{Limitations}

The sample size was not large, although 20 participants per cell were usually regarded as providing high reliability (Thirion et al, 2007). Structural brain abnormalities may contribute to functional alterations, but we did not find significant group differences at a significance level similar to the functional contrast.

It is possible that low or high BMI may be associated with alterations in the cerebral blood flow and fMRI BOLD signal, but little information exists regarding this question. Most studies that have assessed 'resting' brain activity in AN have used SPECT, and found reduced blood perfusion in frontal, parietal, and temporal cortex (Kuruoglu et al, 1998; Rastam et al, 2001). But a recent study found no such abnormalities when a correction for the commonly in AN reduced brain volume was applied (Bailer et al, 2007), suggesting that baseline blood flow is not reduced. In $\mathrm{OB}$, reduced resting blood flow was found in various frontal cortical brain regions (Willeumier et al, 2011), but that study did not correct for brain volume. As OB has been associated with reduced brain GM volumes (Gunstad et al, 2008), reduced regional brain blood flow in OB is not certain, and the found blood-flow reduction may have disappeared if a partial volume correction had been applied, similarly to the results in AN. Furthermore, as blood flow may be rather reduced in both $\mathrm{AN}$ and $\mathrm{OB}$, if anything, such alterations would not explain opposite results in this reward model.

Another possible limitation is that somatosensory response in the AN or OB groups were different compared with $\mathrm{CW}$, and that this may have affected brain response and reward-system activation in the group-by-condition analysis. We did not find somatosensory cortical areas different across groups, suggesting that stimulation in the mouth may not have confounded the results. However, to address this concern more directly, we analyzed the positive-prediction error conditions with the Artificial Saliva receipt as control condition, the negative-prediction error condition with No solution as control, and Sucrose expectation with Artificial Saliva expectation as control condition. Those analyses indicated smaller in size, but still highly significant areas of difference across groups that were consistent with the results in the original analysis. This further suggested that our results were not merely an effect of altered oral stimulation.

Comorbid conditions or medication treatment may have confounded the imaging results in AN. We addressed this by comparing $\mathrm{AN}$ individuals who were without current 
mood or anxiety disorder, or medication with $\mathrm{CW}$ and $\mathrm{OB}$ not on birth control. That comparison indicated similar results compared with the larger three-group comparison for unexpected sucrose receipt or omission, supporting that the findings of the study were not due to medication or comorbid disorder effects. We cannot say whether the results are exclusive to the application of taste or also to non-food reward stimuli, and this will be the focus of future studies. Another potentially confounding factor is the duration of illness on brain function. Both $\mathrm{AN}$ and $\mathrm{OB}$ groups included subjects that had been ill for up to 20 years, but the exact number of months or years sick $v s$ partial recovery and relapse are difficult to reliably quantify. In both groups, duration of illness was not related to brainimaging response, but a prospective study would be better equipped to answer this question. Another limitation is that our results were acquired in groups of ill subjects, and hyper- and hyposensitive reward response could be the state markers of under- and overweight states, whereas it is uncertain whether such reward abnormalities are for instance trait abnormalities in AN. The study by Cowdrey et al (2011) in recovered AN indicated that hypersensitive response to taste stimuli might be present across different states of AN illness. However, a prospective study will be most informative to tease apart the state-related reward system abnormalities from either traits or neurobiological factors that persist long into weight recovery, and that could be vulnerabilities for relapse.

In summary, this study suggests that $\mathrm{AN}$ is associated with heightened, whereas $O B$ is associated with reduced brain reward sensitivity to salient taste stimuli, possibly related to dopamine function. The use of the neurotransmitter model-based tasks and data analysis may have the potential to study those disorders, and could move eating disorders research toward more specific models of altered neurotransmitter system function.

\section{ACKNOWLEDGEMENTS}

We thank Dr O'Doherty for providing the fractal images used as CS in the study. We would like to thank all the individuals who participated in this study, as well as the staff at the Eating Disorders program at the Children's Hospital and the Eating Disorder Center Denver.

\section{DISCLOSURE}

The authors declare no conflict of interest. All authors contributed significantly to this manuscript. Dr Frank is on the scientific advisory board of the Eating Disorders Center of Denver. Dr O'Reilly has received funding through the NIH IBSC center Grant 1-P50-MH079485. A Davis Foundation Award of the Klarman Family Foundation Grants Program in Eating Disorders, and the NIMH Grant K23 MH080135-01A2 provided funding for all aspects of the study to Dr Frank. The following is a list of the funding sources for each of the contributing authors: Dr Frank: NIH 5K23MH080135-04; Dr Reynolds: NIH, MH 079485, P50/IBSC and NIH, R01-DA027748; Dr O'Reilly: iARPA, ICArUS MINDS, NIH IBSC center 1-P50-MH079485 and ONR N00014-10-10177 1/15/10-12/31/12; Dr Yang: NIH 5R01MH085734-02;
Dr Tregellas: VA 1I01CX000459-01 and NIH 5R01DK08909502; Ms Shott And Ms Jappe: not applicable.

\section{REFERENCES}

American Psychiatric Association (2000). Diagnostic and Statistical Manual of Mental Disorders - Text Revision (DSM-IV-TR). Handbook of Psychiatric Measures. American Psychiatric Association: Washington DC.

Anderson JW, Greenway FL, Fujioka K, Gadde KM, McKenney J, O'Neil PM (2002). Bupropion SR enhances weight loss: a 48-week double-blind, placebo- controlled trial. Obes Res 10: 633-641.

Avena NM, Rada P, Hoebel BG (2008). Underweight rats have enhanced dopamine release and blunted acetylcholine response in the nucleus accumbens while bingeing on sucrose. Neuroscience 156: $865-871$.

Bailer UF, Frank GK, Henry SE, Price JC, Meltzer CC, Mathis CA et al (2007). Exaggerated 5-HT1A but normal 5-HT2A receptor activity in individuals ill with anorexia nervosa. Biol Psychiatry 61: 1090-1099.

Barbato G, Fichele M, Senatore I, Casiello M, Muscettola G (2006). Increased dopaminergic activity in restricting-type anorexia nervosa. Psychiatry Res 142: 253-255.

Beck AT, Ward M, Mendelson M, Mock J, Erbaugh J (1961). An Inventory for measuring depression. Arch Gen Psychiatry 4: 53-63.

Berridge KC (2009). Liking' and 'wanting' food rewards: brain substrates and roles in eating disorders. Physiol Behav 97: $537-550$.

Berridge KC, Ho CY, Richard JM, DiFeliceantonio AG (2010). The tempted brain eats: pleasure and desire circuits in obesity and eating disorders. Brain Res 1350: 43-64.

Boettiger CA, Mitchell JM, Tavares VC, Robertson M, Joslyn G, D'Esposito $M$ et al (2007). Immediate reward bias in humans: fronto-parietal networks and a role for the catechol-O-methyltransferase 158(Val/Val) genotype. J Neurosci 27: 14383-14391.

Carmichael ST, Price JL (1996). Connectional networks within the orbital and medial prefrontal cortex of macaque monkeys. J Comp Neurol 371: 179-207.

Carr K, Tsimberg Y, Berman Y, Yamamoto N (2003). Evidence of increased dopamine receptor signaling in food-restricted rats. Neuroscience 119: 1157-1167.

Carr KD (2002). Augmentation of drug reward by chronic food restriction: behavioral evidence and underlying mechanisms. Physiol Behav 76: 353-364.

Carr KD (2007). Chronic food restriction: enhancing effects on drug reward and striatal cell signaling. Physiol Behav 91: 459-472.

Cassano G, Miniati M, Pini S, Rotondo A, Banti S, Borri C et al (2003). Six-month open trial of haloperidol as an adjunctive treatment for anorexia nervosa: a preliminary report. Int J Eat Disord 33: 172-177.

Cloninger C, Przybeck T, Svarkic D, Wetzel R (1994). The Temperament and Character Inventory (TCI): A Guide to Its Development and Use. Center for Psychobiology of Personality, Washington University: St. Louis, MO.

Corsica JA, Pelchat ML (2010). Food addiction: true or false? Curr Opin Gastroenterol 26: 165-169.

Cowdrey FA, Park RJ, Harmer CJ, McCabe C (2011). Increased neural processing of rewarding and aversive food stimuli in recovered anorexia nervosa. Biol Psychiatry 70: 736-743.

D'Ardenne K, McClure SM, Nystrom LE, Cohen JD (2008). BOLD responses reflecting dopaminergic signals in the human ventral tegmental area. Science 319: 1264-1267.

Davis C, Levitan RD, Kaplan AS, Carter J, Reid C, Curtis C et al (2007). Dopamine transporter gene (DAT1) associated with 
appetite suppression to methylphenidate in a case-control study of binge eating disorder. Neuropsychopharmacology 32: 2199-2206.

Daw ND, Doya K (2006). The computational neurobiology of learning and reward. Curr Opin Neurobiol 16: 199-204.

Daw ND, Gershman SJ, Seymour B, Dayan P, Dolan RJ (2011). Model-based influences on humans' choices and striatal prediction errors. Neuron 69: 1204-1215.

de Araujo IE, Ren X, Ferreira JG (2010). Metabolic sensing in brain dopamine systems. Results Probl Cell Differ 52: 69-86.

Dreher JC, Meyer-Lindenberg A, Kohn P, Berman KF (2008). Age-related changes in midbrain dopaminergic regulation of the human reward system. Proc Natl Acad Sci USA 105: 15106-15111.

Dreher JC, Schmidt PJ, Kohn P, Furman D, Rubinow D, Berman KF (2007). Menstrual cycle phase modulates reward-related neural function in women. Proc Natl Acad Sci USA 104: 2465-2470.

Elliott R, Dolan RJ, Frith CD (2000). Dissociable functions in the medial and lateral orbitofrontal cortex: evidence from human neuroimaging studies. Cereb Cortex 10: 308-317.

Felsted JA, Ren X, Chouinard-Decorte F, Small DM (2010). Genetically determined differences in brain response to a primary food reward. J Neurosci 30: 2428-2432.

Fladung AK, Gron G, Grammer K, Herrnberger B, Schilly E, Grasteit $S$ et al (2010). A neural signature of anorexia nervosa in the ventral striatal reward system. Am J Psychiatry 167: 206-212.

Frank GK, Bailer UF, Henry SE, Drevets W, Meltzer CC, Price JC et al (2005). Increased dopamine D2/D3 receptor binding after recovery from anorexia nervosa measured by positron emission tomography and [11c]raclopride. Biol Psychiatry 58: 908-912.

Frank GK, Reynolds JR, Shott ME, O'Reilly RC (2011). Altered temporal difference learning in bulimia nervosa. Biol Psychiatry 70: 728-735.

Garner D (2004). Eating Disorder Inventory ${ }^{\mathrm{TM}}-3$ (EDI $\left.I^{\mathrm{TM}}-3\right)$. Psychological Assessment Resources, Inc.: Lutz, FL.

Gearhardt AN, Yokum S, Orr PT, Stice E, Corbin WR, Brownell KD (2011). Neural correlates of food addiction. Arch Gen Psychiatry 68: $808-816$

Goldfield GS, Lorello C, Doucet E (2007). Methylphenidate reduces energy intake and dietary fat intake in adults: a mechanism of reduced reinforcing value of food? Am J Clin Nutr 86: 308-315.

Goldstein RZ, Tomasi D, Alia-Klein N, Cottone LA, Zhang L, Telang $\mathrm{F}$ et al (2007). Subjective sensitivity to monetary gradients is associated with frontolimbic activation to reward in cocaine abusers. Drug Alcohol Depend 87: 233-240.

Gunstad J, Paul RH, Cohen RA, Tate DF, Spitznagel MB, Grieve S et al (2008). Relationship between body mass index and brain volume in healthy adults. Int J Neurosci 118: 1582-1593.

Halmi KA (2009). Perplexities and provocations of eating disorders. J Child Psychol Psychiatry 50: 163-169.

Hazy TE, Frank MJ, O'Reilly RC (2010). Neural mechanisms of acquired phasic dopamine responses in learning. Neurosci Biobehav Rev 34: 701-720.

Hyman SE, Malenka RC (2001). Addiction and the brain: the neurobiology of compulsion and its persistence. Nat Rev Neurosci 2: 695-703.

Jocham G, Klein TA, Ullsperger M (2011). Dopamine-mediated reinforcement learning signals in the striatum and ventromedial prefrontal cortex underlie value-based choices. J Neurosci 31: 1606-1613.

Johnson PM, Kenny PJ (2010). Dopamine D2 receptors in addiction-like reward dysfunction and compulsive eating in obese rats. Nat Neurosci 13: 635-641.

Karson CN (1983). Spontaneous eye-blink rates and dopaminergic systems. Brain J Neurol 106: 643-653.

Kaye WH, Ebert MH, Raleigh M, Lake R (1984). Abnormalities in CNS monoamine metabolism in anorexia nervosa. Arch Gen Psychiatry 41: 350-355.
Kaye WH, Fudge JL, Paulus M (2009). New insights into symptoms and neurocircuit function of anorexia nervosa. Nat Rev Neurosci 10: $573-584$.

Kelley AE, Baldo BA, Pratt WE, Will MJ (2005). Corticostriatalhypothalamic circuitry and food motivation: integration of energy, action and reward. Physiol Behav 86: 773-795.

Koob GF, Le Moal M (2005). Plasticity of reward neurocircuitry and the 'dark side' of drug addiction. Nat Neurosci 8: 1442-1444.

Kumar P, Waiter G, Ahearn T, Milders M, Reid I, Steele JD (2008). Abnormal temporal difference reward-learning signals in major depression. Brain 131: 2084-2093.

Kuruoglu AC, Kapucu O, Atasever T, Arikan Z, Isik E, Unlu M (1998). Technetium-99m-HMPAO brain SPECT in anorexia nervosa. J Nucl Med 39: 304-306.

Maia TV, Frank MJ (2011). From reinforcement learning models to psychiatric and neurological disorders. Nat Neurosci 14: 154-162.

Menon M, Jensen J, Vitcu I, Graff-Guerrero A, Crawley A, Smith MA et al (2007). Temporal difference modeling of the blood-oxygen level dependent response during aversive conditioning in humans: effects of dopaminergic modulation. Biol Psychiatry 62: 765-772.

Noonan MP, Walton ME, Behrens TE, Sallet J, Buckley MJ, Rushworth MF (2010). Separate value comparison and learning mechanisms in macaque medial and lateral orbitofrontal cortex. Proc Natl Acad Sci USA 107: 20547-20552.

O'Connor R, Colder C, Hawk L (2004). Confirmatory factor analysis of the Sensitivity to Punishment and Sensitivity to Reward Questionnaire. Pers Indiv Differ 37: 985-1002.

O'Doherty JP, Dayan P, Friston K, Critchley H, Dolan RJ (2003). Temporal difference models and reward-related learning in the human brain. Neuron 38: 329-337.

Rastam M, Bjure J, Vestergren E, Uvebrant P, Gillberg IC, Wentz E et al (2001). Regional cerebral blood flow in weight-restored anorexia nervosa: a preliminary study. Dev Med Child Neurol 43: 239-242.

Schultz W (1998). Predictive reward signal of dopamine neurons. J Neurophysiol 80: 1-27.

Schultz W (2002). Getting formal with dopamine and reward. Neuron 36: 241-263.

Schultz W, Dayan P, Montague PR (1997). A neural substrate of prediction and reward. Science 275: 1593-1599.

Small DM, Zatorre RJ, Dagher A, Evans AC, Jones-Gotman M (2001). Changes in brain activity related to eating chocolate: from pleasure to aversion. Brain 124: 1720-1733.

Spielberger CD (1983). Manual for the State-Trate Anxiety Inventory. Consulting Psychologists Press, Inc.: Palo Alto, CA.

Stice E, Yokum S, Blum K, Bohon C (2010). Weight gain is associated with reduced striatal response to palatable food. J Neurosci 30: 13105-13109.

Stoy M, Schlagenhauf F, Sterzer P, Bermpohl F, Hagele C, Suchotzki $\mathrm{K}$ et al (2011). Hyporeactivity of ventral striatum towards incentive stimuli in unmedicated depressed patients normalizes after treatment with escitalopram. J Psychopharmacol; e-pub ahead of print 17 September 2011.

Sutton RS, Barto AG (1998). Toward a Modern Theory of Adaptive Networks: Expectation and Prediction. MIT Press: Boston, MA.

Thirion B, Pinel P, Meriaux S, Roche A, Dehaene S, Poline JB (2007). Analysis of a large fMRI cohort: statistical and methodological issues for group analyses. Neuroimage 35: 105-120.

Trunko ME, Schwartz TA, Duvvuri V, Kaye WH (2011). Aripiprazole in anorexia nervosa and low-weight bulimia nervosa: case reports. Int J Eat Disord 44: 269-275.

Tsujimoto S, Genovesio A, Wise SP (2009). Monkey orbitofrontal cortex encodes response choices near feedback time. J Neurosci 29: 2569-2574.

Volkow ND, Tomasi D, Wang GJ, Fowler JS, Telang F, Goldstein $\mathrm{RZ}$ et al (2011). Positive emotionality is associated with baseline 
metabolism in orbitofrontal cortex and in regions of the default network. Mol Psychiatry 16: 818-825.

Volkow ND, Wang GJ, Telang F, Fowler JS, Thanos PK, Logan J et al (2008). Low dopamine striatal D2 receptors are associated with prefrontal metabolism in obese subjects: possible contributing factors. Neuroimage 42: 1537-1543.

Wagner A, Aizenstein H, Mazurkewicz L, Fudge J, Frank GK, Putnam K et al (2008). Altered insula response to taste stimuli in individuals recovered from restricting-type anorexia nervosa. Neuropsychopharmacology 33: 513-523.
Wagner A, Aizenstein H, Venkatraman VK, Fudge J, May JC, Mazurkewicz L et al (2007). Altered reward processing in women recovered from anorexia nervosa. Am J Psychiatry 164: 1842-1849.

Waltz JA, Schweitzer JB, Ross TJ, Kurup PK, Salmeron BJ, Rose EJ et al (2010). Abnormal responses to monetary outcomes in cortex, but not in the basal ganglia, in schizophrenia. Neuropsychopharmacology 35: 2427-2439.

Willeumier KC, Taylor DV, Amen DG (2011). Elevated BMI is associated with decreased blood flow in the prefrontal cortex using SPECT imaging in healthy adults. Obesity 19: 1095-1097.

Supplementary Information accompanies the paper on the Neuropsychopharmacology website (http://www.nature.com/npp) 